\title{
Quantification of Intra-hour Security-constrained Flexibility Region
}

\author{
Zhijun Qin, Member, IEEE, Yunhe Hou, Senior Member, IEEE, Shunbo Lei, Student Member, IEEE, and \\ Feng Liu, Member, IEEE
}

\begin{abstract}
Rapid growth of renewable energy sources (RES) in the generation capacity mix poses substantial challenges on the operation of power systems in various time scales. Particularly in the intra-hour time scale, the interplay among variability and uncertainty of RES, unexpected transmission/generation outages, and short dispatch lead time cause difficulties in generationload balancing. This paper proposes a method to quantify the intra-hour flexibility region. A robust security-constrained multiperiod optimal power flow (RSC-OPF) model is first constructed to quantify the frequency, magnitude, and intensity of insufficient flexibility. The randomness of RES is captured by uncertainty sets in this model. The N-k contingency, spinning reserve, and corrective control limit constraints are included. This model is then cast into a two-stage robust optimization (RO) model and solved by the column-and-constraint generation (C\&CG) method. The emergency measures with a least number of affected buses are derived and subsequently assessed by the post-optimization sensitivity analysis. Finally, the operational flexibility region is determined by continuous perturbation on the RES penetration level and the forecast error. The IEEE 14-bus system and a realistic Chinese 157-bus system are used to demonstrate the proposed method.
\end{abstract}

Index Terms-Power system flexibility, robust optimization, DC power flow, optimal power flow, contingency analysis.

\section{NOMENCLATURE}

$$
\begin{gathered}
\text { A. Variables } \\
I_{i, t, c} \\
\Delta P_{i, t, c}^{\text {curt }} \\
\Delta P_{i, t, c}^{\text {shed }} \\
P_{i, t, c}^{\text {gen }} \\
\theta_{i, t, c} \\
\tau_{i, t, c}
\end{gathered}
$$

Imbalance state of bus $i$ at time interval $t$ on contingency $c$ ( $c=0$ denotes the base case).

Generation curtailment on bus $i$ at time interval $t$ on contingency $c$.

Load shedding on bus $i$ at time interval $t$ on contingency $c$.

Output of dispatchable generator on bus $i$ at time interval $t$ on contingency $c$.

Phase angle of bus $i$ at time interval $t$ on contingency $c$.

Effective ramping time of dispatchable generator on bus $i$ at time interval $t$ on contingency $c$ to provide spinning reserve.

This research is supported by National Basic Research Program of China (973 Program, 2012CB215102), Research Grant Council (RGC), Hong Kong SAR, under grant GRF17202714 and ECS739713, RGC Theme-based Research Scheme (TRS) under grant T23-701/ 14-N, and National Natural Science Foundation of China under grant 51277155.

Zhijun Qin, Yunhe Hou and Shunbo Lei are with the Department of Electrical and Electronic Engineering, The University of Hong Kong (HKU), Hong Kong SAR, China, e-mail: (zjqin@eee.hku.hk, yhhou@eee.hku.hk, leishunbo@eee.hku.hk). Yunhe Hou is also with HKU Shenzhen Institute of Research and Innovation, Shenzhen, China. Feng Liu is with the State Key Laboratory of Power Systems, Department of Electrical Engineering, Tsinghua University, Beijing, China (e-mail: lfeng@mail.tsinghua.edu.cn).

\section{B. Parameters}

$P_{i}^{\mathrm{g}, \max } \quad$ Maximum output of dispatchable generator on bus $i$.

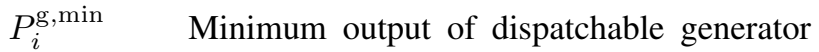
on bus $i$.

$R U_{i} \quad$ Ramping rate of dispatchable generator on bus $i$ to provide spinning reserve.

$\Delta P_{i}^{\mathrm{g}, \text { up }} \quad$ Ramping up capacity of dispatchable generator on bus $i$.

$\Delta P_{i}^{\mathrm{g}, \mathrm{dn}} \quad$ Ramping down capacity of dispatchable generator on bus $i$.

$P_{i j}^{\max } \quad$ Transmission limit of the branch between bus $i$ and bus $j$.

$E_{i}^{\text {gen }}, \varepsilon_{i} \quad$ The limit of total generation energy and the corresponding tolerance of bus $i$.

$\Delta P_{i}^{\mathrm{re}, \max }$ Maximum contingency reserve of dispatchable generator on bus $i$.

$B_{i j, c}^{\text {bus }} \quad$ Bus admittance matrix element between bus $i$ and bus $j$ on contingency $c$.

$B_{i j, c}^{\text {branch }} \quad$ Branch admittance between bus $i$ and bus $j$ on contingency $c$.

$\Delta P_{i}^{\mathrm{im}, \max }$ Maximum power imbalance on bus $i$.

$\tau^{\max } \quad$ Maximum ramping time for dispatchable generators to provide spinning reserve, typically 10-15 minutes.

$p_{c} \quad$ Probability of transmission contingency $c$.

C. Random Variable and Uncertainty Set

$\tilde{P}_{i . t}^{\text {load }} \quad$ Net load on bus $i$ at time interval $t$.

$P_{i . t}^{\text {load,max }}$ Maximum net load on bus $i$ at time interval $t$.

$P_{i . t}^{\text {load,min }}$ Minimum net load on bus $i$ at time interval $t$.

D. Numbers and Sets

$N B \quad$ Number of buses.

NT Number of time intervals.

NC Number of contingencies.

$N W \quad$ Number of wind farms.

NL Number of load with uncertain demand.

$\mathscr{G}(t) \quad$ Set of online generators at time interval $t$. 


\section{INTRODUCTION}

$\mathbf{T}$ $\mathrm{O}$ achieve more sustainable and competitive economics, many economic entities have made tremendous efforts towards increasing the share of renewable energy sources (RES) in their generation mix. Fast substitution of dispatchable generation capacity with RES has imposed considerable challenges on the planning and operation of power systems. The salient features of RES, namely variability and uncertainty [1], cause substantial challenges in generation-load balancing and effective utilization of the renewable energy. These challenges lead to an emerging research area concerning power system flexibility, which has attracted much research efforts [2], [3].

The uncertainty and variability of RES present different characteristics over various time scales [1]. The time scale separation methodology does not thoroughly consider the significant variability within various time resolutions, therefore may cause generation-load balancing issues. Especially, the intra-hour variability will be an important issue when the RES penetration level reaches a certain point (see Part II, Grid Integration in [4]).

This paper aims to propose a method to quantify the intrahour flexibility, considering the interplay among the uncertainty and the variability of RES, transmission contingencies, loss of energy contingencies, and operational constraints of dispatchable generators due to the short dispatch lead time.

\section{A. Related Work}

It is demonstrated that the uncertainty and variability of RES, the fluctuation of load demand, together with the unexpected contingencies drive the need for greater power system flexibility [3].

1) Flexibility resources:

The provision of flexibility comes from institutional enablers (such as market design [3] and interconnection [5]), flexible generation technology [6], energy storage [7], [8], spinning reserves [9], and additional sources of flexibility (e.g., electric vehicles [10] and demand response (DR) [11], [12]). Especially, hourly DR program is beneficial in reducing the cost of security-based power system scheduling [13], in lowering load payment [14], and in improving the thermal generation flexibility [15]. DR program in various time scales also contributes to the load-following reserve [16] and contingency reserve under high RES penetration. The time-flexible demand [17] and demand response [18] have been applied to tackle intra-hour load/wind variation and unexpected outages.

\section{2) Definition and metrics:}

Various definitions and metrics have been proposed to study the need for, and the provision of power system flexibility, ranging from long-term planning [19], [20] to short-term operation [21]. Power system flexibility can be studied from insufficiency, provision, and availability perspectives.

In [22], flexibility is defined as "the ability of a system to deploy its resources to respond to changes in net load". The insufficient ramping resource expectation (IRRE) is proposed to quantify the flexibility in long-term planning. The lack of ramp probability (LORP) is defined as an operational flexibility metric in [23] and quantified using a two-step robust optimization (RO) based framework.
In [24], the flexibility describes "the ability of a power system to cope with variability and uncertainty over various time horizons." The provision of adjustable capacity and ramping capacity over a given time interval is defined as an operational flexibility index. The work in [25] extends this flexibility index as the generation-characteristics-based (GCB) flexibility, and quantifies the GCB index using an optimization model, considering the transmission constraints with power transfer distribution factors (PTDFs).

The availability of operational flexibility can be studied via the existence of feasible operation strategies. In [5] and [26], operational flexibility is the ability of the power system to react to a disturbance to keep the system secure. The available flexibility, considering the ramping, capacity, and energy of dispatchable generators, transmission constraints in DC power flow, and $\mathrm{N}-1$ reliability criterion, is described as a polyhedron entitled "flexibility set", or "reach set" in [27]. The work in [28], [29] and [30] construct a flexibility metric using the RO models to reflect the largest range of uncertainty that a power system can handle, and the do-not-exceed (DNE) limits of RES range. By contrast, [31] defines flexibility as trackability, namely the ability to track any realization of the net load random process using a probabilistic approach.

3) Modeling and solution methodology:

In view of the spatial-temporal-dependency of the uncertainty and variability of RES, comprehensive inter-temporal simulations [22], Monte Carlo Simulation [12], and optimization methods [32], [33] are applied to study the flexibility requirements in long-term expansion planning and in shortterm reserve planning. Modeling the dynamics of dispatchable components using the state-space representation, the flexibility envelops are calculated using the reach set approach [27], [34], or a parametric approach [35].

\section{B. Contributions of this paper}

As the transmission infrastructure and flexible generation resources supporting high RES penetration cannot be readily put in place, the surge in RES installation imposes stress in the operation of legacy power systems. Therefore, this paper places the emphasis on operational flexibility assessment. Specifically, the objective of this paper is to study the evolution of power systems from available flexibility to insufficient flexibility, driven by ever-changing system parameters (such as the RES penetration level and the associated forecast error).

To this end, the essential challenge is to investigate operational flexibility from a coherent perspective for both availability and insufficiency within one methodology, considering the interplay among uncertainty and variability of RES, and the unexpected outages of transmission lines or generators.

The contributions of this paper are summarized below.

1) This paper proposes a robust security-constrained multiperiod optimal power flow (RSC-OPF) model to quantify intra-hour operational flexibility considering the $\mathrm{N}-\mathrm{k}$ security criterion as well as spinning reserves and contingency reserve constraints of dispatchable generators. The objective value of this model indicates the worstcase expectation of total reluctant load shedding and/or wind curtailment over the studied time horizon. The 
worst-case combination of the unexpected outage and the wind profile over the studied time horizon is also identified. In case that insufficient flexibility occurs due to insufficient reserve from dispatchable generators, the output of the proposed model can provide the amount of reserve requirement for various flexible resources, including but not limited to demand response, energy storage, and quick-start units.

2) The results obtained by the proposed model can be used to construct various quantitative flexibility metrics. For example, despite that the frequency, magnitude, and intensity of insufficient flexibility are proposed by North America Electric Reliability Corporation (NERC) [2], these metrics are not quantitatively defined. In this paper, these metrics are quantified by the proposed model. Meanwhile, the effectiveness of emergency measures is obtained from a post-optimization sensitivity analysis.

3) The intra-hour operational flexibility region is proposed to describe the maximum/minimum RES penetration level and the associated forecast error that a power grid can handle, with the limited dispatchable resources in a short dispatch lead time. The operational flexibility region is determined in terms of the flexibility metrics (obtained by the proposed model) over the space spanned by as the RES penetration level and the associated forecast error. The visualized flexibility region helps system operators study the impact of the aforementioned system parameters on operational flexibility.

The proposed model shares some similarities with the security-constrained unit commitment (SCUC) and the stochastic multi-period OPF [36]. For sake of clarification, the fundamental differences between the proposed model and related work are listed below.

1) The objectives of SCUC and the proposed model are different. The objective of SCUC is to determine the (typically hourly) unit commitment schedule with minimum commitment and dispatch cost, considering various operational constraints and transmission/generation contingencies. The uncertainty of RES, particularly wind power, can be characterized by certain probability distributions (stochastic SCUC, e.g., [37]), deterministic uncertainty sets (Robust SCUC, e.g., [38], [39]), or hybrid of these two formulations [40]. By contrast, the objective of RSC-OPF is to determine the intra-hour emergency measures, if necessary, with minimum total amount of reluctant load shedding and wind curtailment in the worst case, given the hourly on/off states of generating units as known parameters.

2) The dispatch lead time and the time resolutions between SCUC and the proposed model are different. The dayahead SCUC considers hourly unit commitment. Lookahead UC will be applied to bridge the SCUC and real-time economic dispatch for incorporating largescale intermittent RES. However, RSC-OPF considers the operational flexibility within the intra-hour time scale, which leads to a much shorter dispatch lead time and very limited dispatchable resources. Therefore, it is necessary to scrutinize the operational constraints vigorously. Particularly, unlike a pre-specified reserve level (such as $20 \%$ ) or a constant reserve bound (e.g., SCUC and [18], [16]), the spinning reserve constraints on dispatchable generators in RSC-OPF scan each loss of energy contingency to guarantee the system reliability.

3) The transmission contingencies are processed in different fashion in SCUC and in RSC-OPF. SCUC consists of a master UC sub-problem, a base case network security evaluation sub-problem and a number of contingency network security evaluation sub-problems [41]. In SCUC, transmission contingencies are checked for each single hour, independently. By contrast, in RSC-OPF, the transmission contingency will last for a time horizon. In this studied time horizon, the limited contingency reserve along with the uncertainty and variability of RES output will impose great stress in power system operation. The interaction between contingencies and RES output over the studied time horizon is studied by the RSC-OPF model, with consideration on the timerelated operational constraints and limited contingency reserve.

4) The salient feature of this work is the combination of the probabilistic nature of the contingencies and the deterministic uncertainty set to describe the uncertain nature of RES output in the intra-hour time scale. Different from the scenario-base methods (e.g., [37], [17], [18], [16]), the proposed model is able to identify the worstcase combination of intra-hour RES output realization and the most severe contingency, which together lead to insufficient flexibility.

5) To focus on the proposed method in assessing the operational flexibility, the load-following reserve and the contingency reserve come from dispatchable generators in this paper. However, the proposed method can be extended to consider the contribution of various flexible resources in operational flexibility, as to be discussed in Section II.

The rest of this paper is organized as follows. The proposed RSC-OPF model is formulated as a two-stage RO model in Section II. The $1^{\text {st }}$ stage minimizes the number of power imbalance events over the time horizon, i.e., the frequency of insufficient flexibility. The $2^{\text {nd }}$ stage minimizes the magnitude of total power imbalance over the time horizon, i.e., the magnitude of insufficient flexibility. In Section III, the proposed RSC-OPF model is solved by the column-and-constraint generation (C\&CG) algorithm [42]. Inspired by [43], a modified Benders Decomposition (BD) procedure is derived to reduce the computational complexity introduced by the contingency list. The case studies are provided in Section IV. Finally, this paper is concluded in Section V.

\section{Model Formulation and Flexibility Metrics}

\section{A. Problem Statement and Assumptions}

This study investigates intra-hour operational flexibility over a time horizon of $\leq 24$ hours. The operational flexibility is studied via the existence of feasible operation strategies to avoid reluctant load shedding and/or wind curtailment, subject 
to comprehensive operational constraints. The power system can be described as flexible if, in the worst-case realization of uncertainty from both RES and unexpected outages, a feasible operation strategy over the time horizon can be identified. The amounts of load shedding and wind curtailment are introduced as slack variables in the power balance constraint, making the power balance as soft constraints.

The assumptions to formulate the model are listed below.

1) The hourly day-ahead unit commitment is predetermined and is regarded as the input for the intra-hour operational flexibility assessment.

2) It has been demonstrated [32] that non-parametric representation is suitable to describe the intra-hour deviation of wind outputs. The uncertainty of the net load is therefore described using the uncertainty set.

3) The unexpected outage of transmission lines affects the entire time horizon.

\section{B. Mathematical Formulation}

The RSC-OPF model for operational flexibility assessment is formulated as (1)-(9). The objective function (1) is to minimize the worst-case expectation of total power imbalance (load shedding and wind curtailment as the corresponding emergency measures) of the power system over a time horizon. For each time interval under each contingency, the operational constraints include power balance (2)-(3), generator output limits (4), and transmission limits (5). The uncertainty of net load of each bus is modeled as an uncertainty set in (3a). For example, for an uncertain load demand with the mean value as $30 \mathrm{MW}$ and $3 \%$ forecast error, the associated uncertainty set is $[29.1,30.9]$ MW. To safeguard the loss of energy contingencies, the spinning reserve (6) is taken into considerations. In the presence of any loss of energy contingency, the survival units should ramp up to a given period $\tau^{\max }$ to compensate the lost generation capacity. Note that the effective spinning reserve (determined by $\tau_{i, t, c} R U_{i}$ ) should not exceed the available reserve (see e.g., [44]). The dispatchable generators are subject to ramping rate limits (7). Some dispatchable generators are supposed to deliver certain energy (8) due to bilateral contract constraints or fuel constraints (e.g., natural gas contract and reservoir capacity). Particularly, in the presence of transmission contingencies, the adjustment of each dispatchable generator for corrective control is limited to the corresponding contingency reserve (9) (see e.g., [37], [45]) due to the short dispatch lead time. Note that the spinning up/down reserve to hedge the uncertainty of the net load (3a) has been determined by (4) and (7).

1) Objective function:

$$
\max \min \left(\sum_{i=1}^{N B} \sum_{t=1}^{N T} \sum_{c=0}^{N C} p_{c} I_{i, t, c}\left(\Delta P_{i, t, c}^{\text {curt }}+\Delta P_{i, t, c}^{\text {shed }}\right)\right)
$$

2) Operational constraints $\forall i, \forall t, \forall c$ :

$$
P_{i, t, c}^{\mathrm{gen}}-\sum_{j=1}^{N B} B_{i j, c}^{\mathrm{bus}} \theta_{j, t, c}+I_{i, t, c}\left(\Delta P_{i, t, c}^{\mathrm{shed}}-\Delta P_{i, t, c}^{\mathrm{curt}}\right)=\tilde{P}_{i, t}^{\mathrm{load}}
$$

$$
\begin{gathered}
\tilde{P}_{i, t}^{\text {load }} \in\left[P_{i . t}^{\text {load }, \text { min }}, P_{i . t}^{\text {load }, \max }\right] \\
\Delta P_{i, t, c}^{\text {shed }} \geq 0, \Delta P_{i, t, c}^{\text {curt }} \geq 0 \\
I_{i, t, c} \in\{0,1\} \\
\forall i \in \mathscr{G}(t), P_{i}^{\mathrm{g}, \min } \leq P_{i, t, c}^{\mathrm{gen}} \leq P_{i}^{\mathrm{g}, \max } \\
-P_{i j}^{\max } \leq B_{i j, c}^{\text {branch }}\left(\theta_{i, t, c}-\theta_{j, t, c} \leq P_{i j}^{\max }\right. \\
\forall i, j \in \mathscr{G}(t), P_{i, t, c}^{\mathrm{gen}}+\tau_{i, t, c} R U_{i} \leq P_{i}^{\mathrm{g} . \max } \\
0 \leq \tau_{i, t, c} \leq \tau^{\max } \\
P_{i, t, c}^{\mathrm{gen}} \leq \sum_{j \neq i}\left(\tau_{j, t, c} R U_{j}\right)
\end{gathered}
$$

3) Inter-temporal constraints:

$$
\begin{gathered}
-\Delta P_{i}^{\mathrm{g}, \mathrm{dn}} \leq P_{i, t, c}^{\mathrm{gen}}-P_{i,(t-1), c}^{\mathrm{gen}} \leq \Delta P_{i}^{\mathrm{g}, \mathrm{up}}, \\
\forall i \in \mathscr{G}(t) \cap \mathscr{G}(t-1), \forall t, \forall c \\
E_{i}^{\mathrm{gen}}-\varepsilon_{i} \leq \sum_{t=1}^{N T} P_{i, t, c}^{\mathrm{gen}} \leq E_{i}^{\mathrm{gen}}+\varepsilon_{i}, \forall i, \forall c
\end{gathered}
$$

4) Corrective control limits $\forall t, \forall c>0$ :

$$
-\Delta P_{i}^{\mathrm{re}, \max } \leq P_{i, t, c}^{\mathrm{gen}}-P_{i, t, 0}^{\mathrm{gen}} \leq \Delta P_{i}^{\mathrm{re}, \max }, \forall i \in \mathscr{G}(t)
$$

Note that the objective function (1) and power balance (2) contain the product of binary variables and continuous variables. By introducing a non-trivial bound $\Delta P_{i}^{\mathrm{im}, \max }$, the amount of power imbalance of each bus is governed by the inequality constraints (10). The objective function and power balance can therefore be rewritten as (11) and (12), respectively.

$$
\begin{gathered}
0 \leq \Delta P_{i, t, c}^{\text {shed }} \leq \Delta P_{i}^{\mathrm{im}, \max } I_{i, t, c} \\
0 \leq \Delta P_{i, t, c}^{\mathrm{curt}} \leq \Delta P_{i}^{\mathrm{im}, \max } I_{i, t, c} \\
\max \min \left(\sum_{i=1}^{N B} \sum_{t=1}^{N T} \sum_{c=0}^{N C} p_{c}\left(\Delta P_{i, t, c}^{\text {curt }}+\Delta P_{i, t, c}^{\text {shed }}\right)\right) \\
P_{i, t, c}^{\text {gen }}-\sum_{j=1}^{N B} B_{i j, c}^{\text {bus }} \theta_{j, t, c}+\left(\Delta P_{i, t, c}^{\text {shed }}-\Delta P_{i, t, c}^{\text {curt }}\right)=\tilde{P}_{i, t}^{\text {load }}
\end{gathered}
$$

Remark 1:The decision variable $I_{i . t . c}$ determines the power imbalance state of bus $i$ at time interval $t$ on contingency $c$. The non-trivial $\Delta P_{i}^{\mathrm{im}, \max }$ can be configured to prevent an unrealistically large power imbalance occurring in a particular bus (see Section IV C), or to prevent power imbalance occurring in connection buses (without load or generation).

In summary, the proposed RSC-OPF model is given as follows.

$$
\begin{array}{r}
\max \min \left(\sum_{i=1}^{N B} \sum_{t=1}^{N T} \sum_{c=0}^{N C} p_{c}\left(\Delta P_{i, t, c}^{\text {exce }}+\Delta P_{i, t, c}^{\text {insuf }}\right)\right) \\
\text { s.t. }(10),(12),(3)-(9)
\end{array}
$$


Remark 2: The emergency measures (i.e., the recourse actions) considered in the proposed model are load shedding and wind curtailment. Other resources that improve the operational flexibility, such as demand response, quick-start units, and energy storage, can also be considered in the intra-hour operational flexibility assessment. To this end, the proposed model can be extended to incorporate the demand response (DR) program model (see e.g., [13], [14]), the energy storage model (e.g., [46]), and the quick-start unit model [43]. However, the discrete DR bid scheme and the quickstart unit as the recourse actions will lead to a mixed-integer model in the $2^{\text {nd }}$ stage of the proposed RO model. A nested $C \& C G$ algorithm [43] will be applied to solve the proposed model with discrete DR bid scheme and quick-start units. Note that the hourly DR and quick-start unit model should be extended into intra-hour time scale, which will also increase the computational complexity.

\section{Quantification of Flexibility Metrics}

The results obtained by the proposed model provide the worst-case total power imbalances due to insufficient flexibility. Various quantitative metrics can therefore be constructed based on these results. For example, inspired by [2], the frequency of insufficient flexibility (FIF) can be defined as the expectation of the number of insufficient flexibility events over a time horizon. The magnitude of insufficient flexibility (MIF) can be defined as the expectation of total power imbalance (load shedding and wind curtailment) over the time horizon. The intensity of insufficient flexibility (IIF) can be defined as the average power imbalance per event. Accordingly, FIF, MIF, and IIF are calculated as follows.

$$
\begin{gathered}
\mathrm{FIF}=\left(\sum_{i=1}^{N B} \sum_{t=1}^{N T} \sum_{c=0}^{N C} p_{c} I_{i . t . c}\right) / N T \\
\mathrm{MIF}=\left(\sum_{i=1}^{N B} \sum_{t=1}^{N T} \sum_{c=0}^{N C} p_{c}\left(\Delta P_{i, t, c}^{\text {exce }}+\Delta P_{i, t, c}^{\text {insuf }}\right)\right) \\
\mathrm{IIF}=\operatorname{MIF} /\left(\sum_{i=1}^{N B} \sum_{t=1}^{N T} \sum_{c=0}^{N C} p_{c} I_{i . t . c}\right)
\end{gathered}
$$

Different from those metrics focusing on realizable ramping capacity (e.g., [20], [22]), these metrics represent the worstcase expectation of total amount of emergency measures in the presence of various generation/transmission contingencies, due to the violation of comprehensive operational constraints, including but not limited to capacity and ramping constraints. Therefore, these metrics are robust (therefore conservative) against all possible realization of RES output.

\section{Solution Methodology}

The corrective control limits in (9) introduce coupling between the base case and other contingencies. In this section, the C\&CG algorithm is first applied to perform flexibility assessment for each contingency, including the base case. Then the modified Benders Decomposition (BD) procedure is derived to solve the entire model.

\section{A. $C \& C G$ algorithm to assess flexibility for each contingency}

Once (9) is removed, the flexibility assessment for each contingency can be performed independently. The proposed model associated with each contingency can be cast into a compact form as

$$
\begin{array}{ll} 
& \max \min \boldsymbol{b}^{T} \boldsymbol{y} \\
\text { s.t. } & \boldsymbol{x} \in\{0,1\}^{n} \\
& \boldsymbol{H} \boldsymbol{y} \geq \boldsymbol{h} \\
& \boldsymbol{N} \boldsymbol{y}=\tilde{\boldsymbol{d}} \\
& \boldsymbol{A} \boldsymbol{x}+\boldsymbol{B} \boldsymbol{y} \geq \boldsymbol{g} \\
& \tilde{\boldsymbol{d}} \in\left[\boldsymbol{d}_{\min }, \boldsymbol{d}_{\max }\right]
\end{array}
$$

where $\boldsymbol{x}$ denotes $I_{i, t, c}, \boldsymbol{y}$ denotes the rest decision variables, (17b) denotes (3c), (17c) denotes (3b), (4)-(8), (17d) denotes (12), (17e) denotes (10), and (17f) denotes (3a).

The basic idea of the C\&CG algorithm for solving twostage RO models is to identify the worst-case realizations of uncertainty gradually. Given a set of identified realization of uncertainty $\boldsymbol{D}=\left\{\boldsymbol{d}_{1}, \boldsymbol{d}_{2}, \ldots \boldsymbol{d}_{k}\right\}$, the master problem (MP) associated with (17) is formulated as below.

$$
\begin{array}{ll} 
& \min _{\boldsymbol{x}, \boldsymbol{y}_{l}, \eta} \eta \\
\text { s.t. } & \boldsymbol{x} \in\{0,1\}^{n} \\
& \eta \geq 0 \\
& \eta \geq \boldsymbol{b}^{T} \boldsymbol{y}_{l}, \forall l \leq k \\
& \boldsymbol{H} \boldsymbol{y}_{l} \geq \boldsymbol{h}, \forall l \leq k \\
& \boldsymbol{N} \boldsymbol{y}_{l}=\boldsymbol{d}_{l}, \forall l \leq k \\
& \boldsymbol{A x}+\boldsymbol{B} \boldsymbol{y}_{l} \geq \boldsymbol{g}, \forall l \leq k
\end{array}
$$

Note that system operators may be interested in a few buses with large power imbalance (high intensity inflexibility event), rather than a number of buses with minor power imbalance (low intensity inflexibility event). Therefore, (18a) is reformulated to minimize the total number of buses with power imbalance, given by $\boldsymbol{e}^{T} \boldsymbol{x}+\eta$, where $\boldsymbol{e}$ is an array of 1 with the compatible size. As a result, system operators are able to derive emergency measures (load shedding and/or wind curtailment) on a limited number of affected buses. Therefore, the MP associated with (17) is given by

$$
\begin{array}{cc}
(\mathbf{M P}) & \min _{\boldsymbol{x}, \boldsymbol{y}_{l}, \eta} \boldsymbol{e}^{T} \boldsymbol{x}+\eta \\
\text { s.t. } & (18 \mathrm{~b})-(18 \mathrm{~g})
\end{array}
$$

With the optimal solution $\left(\boldsymbol{x}^{*}, \boldsymbol{y}_{l}^{*}, \eta^{*}\right)$ obtained by solving (19), the subproblem (SP) associated with (17) is given by

$$
\begin{aligned}
\text { (SP) } & Q\left(\boldsymbol{x}^{*}\right)=\max _{\tilde{\boldsymbol{d}}} \min _{\boldsymbol{y}} \boldsymbol{b}^{T} \boldsymbol{y} \\
\text { s.t. } & \boldsymbol{N} \boldsymbol{y}=\tilde{\boldsymbol{d}} \\
& \boldsymbol{M} \boldsymbol{y} \geq \boldsymbol{m} \\
& \tilde{\boldsymbol{d}} \in\left[\boldsymbol{d}_{\min }, \boldsymbol{d}_{\max }\right]
\end{aligned}
$$

where $\boldsymbol{M}=[\boldsymbol{H} ; \boldsymbol{B}], \boldsymbol{m}=\left[\boldsymbol{h} ; \boldsymbol{g}-\boldsymbol{A} \boldsymbol{x}^{*}\right]$. Here, [;] is the vertical concatenation of two matrices or vectors. 
Note that the SP in (20) should either yield a new worstcase realization of $\hat{d}$ if $x^{*}$ leads to a feasible SP, otherwise should generate feasibility cuts for the MP. By merging the dual of the inner minimization problem, (20) can be cast into a non-convex bilinear programming model, and subsequently cast into a mixed-integer linear programming (MILP) model (see detail in the Appendix).

To summarize, the C\&CG algorithm to solve (17) is described as in Algorithm 1. Notice that, if $\hat{\boldsymbol{d}}$ is an infeasible realization given $\boldsymbol{x}^{*}$, a new instance $\hat{\boldsymbol{d}}$ will be inserted in the non-empty set $\boldsymbol{D}$. As a result, a feasibility cut $(18 \mathrm{~g})$ will be generated with this new instance. Subsequently, a change in $\boldsymbol{x}$ will be enforced by re-solving (19). Assuming the solution of SP can be obtained from an oracle, the convergence of the C\&CG algorithm is proven in [42].

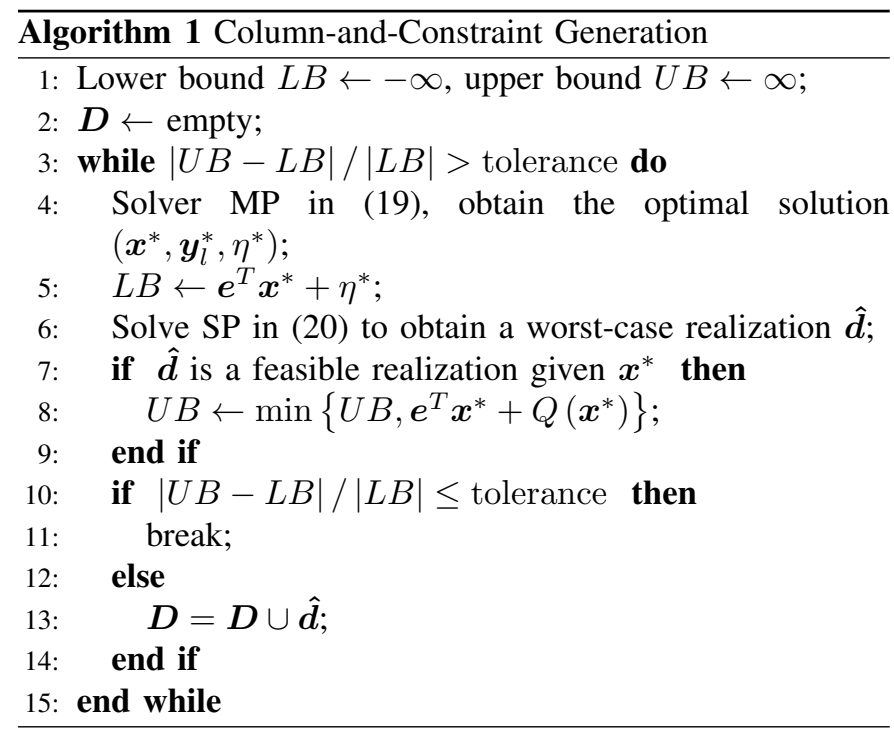

\section{B. Modified BD considering contingencies}

Note that the power balance in (12) is generally satisfied owing to the variables $\Delta P_{i, t, c}^{\text {exce }}$ and $\Delta P_{i, t, c}^{\text {insuf }}$ (if the $\Delta P_{i}^{\text {im,max }}$ is sufficiently large). The difference between [43] and this work is that an optimality cut is generated other than the feasibility cut in [43]. Note that the RO model is a MILP and not applicable for dualization. Therefore, we generate optimality cuts in (17) to reduce the MIF in the objective function, with the obtained $1^{\text {st }}$ stage decision variable and the worst-case realization. Accordingly, the procedure of modified BD to solve the proposed model is as follows.

1) Remove constraint (9) from the base case, solve the following relaxed base case sub-model using Algorithm 1 , and obtain $P_{i, t, 0}^{\text {gen }}$.

$$
\max \min \left(\sum_{i=1}^{N B} \sum_{t=1}^{N T}\left(p_{0} \Delta P_{i, t, 0}^{\text {curt }}+\Delta P_{i, t, 0}^{\text {shed }}\right)\right)
$$

$$
\text { s.t. }(10),(12),(3)-(8)
$$

2) Reformulate (9) into

$$
\begin{aligned}
P_{i, t, c}^{\text {gen }} \geq & P_{i, t, 0}^{\text {gen }}-\Delta P_{i}^{\mathrm{re}, \max } \\
-P_{i, t, c}^{\mathrm{gen}} \geq & -P_{i, t, 0}^{\mathrm{gen}}-\Delta P_{i}^{\mathrm{re}, \max }
\end{aligned}
$$

Use Algorithm 1 to solve the following contingency submodel for each contingency $c$, independently.

$$
\max \min \left(\sum_{i=1}^{N B} \sum_{t=1}^{N T} p_{c}\left(\Delta P_{i, t, c}^{\text {curt }}+\Delta P_{i, t, c}^{\text {shed }}\right)\right)
$$

$$
\text { s.t. (10), (12), (3) - (8), (22) }
$$

3) For each contingency $c>0$, solve (23) with the $1^{\text {st }}$ stage decision variable and identified worst-case realizations (therefore (23) degrades to a deterministic linear programming model), and obtain the local Lagrangian multiplier $\pi_{c}$ associated with (22). Generate the optimality cut for all contingencies using

$$
\alpha \geq \sum_{c=1}^{N C} \pi_{c}\left[P_{i, t, 0}^{\mathrm{gen}}-\Delta P_{i}^{\mathrm{re}, \max } ;-P_{i, t, 0}^{\mathrm{gen}}-\Delta P_{i}^{\mathrm{re}, \max }\right]
$$

with the binding constraints in (22).

4) Use Algorithm 1 to solve the following base case submodel

$$
\max \min \left(\alpha+\sum_{i=1}^{N B} \sum_{t=1}^{N T} p_{0}\left(\Delta P_{i, t, 0}^{\text {exce }}+\Delta P_{i, t, 0}^{\text {insuf }}\right)\right)
$$

s.t. $(10),(12),(3)-(8),(24)$

5) If the change of the objective value (25a) is less than $1 \%$, stop; otherwise go to 2 ).

The relationships among the aforementioned sub-models are shown in Fig.1. Instead of solving the original model, we use C\&CG algorithm to solve the contingency sub-models and base case sub-model within the Benders iteration, in order to reduce the computational complexity. We want to remark that the modified $\mathrm{BD}$ cuts may not theoretically guarantee the global optimality since the RSC-OPF is a mixed-integer model. However, the modified BD cuts have been successfully applied in similar applications, such as stochastic SCUC [37] and robust SCUC [43].

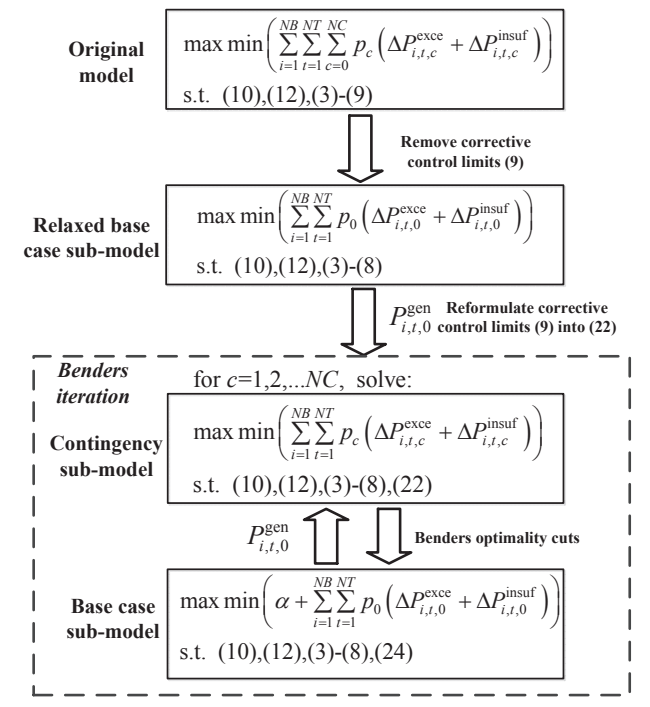

Fig. 1. Relationship among sub-models in Benders iteration 


\section{Computational complexity analysis}

As the BD process decomposes the base case sub-model and the contingency sub-models, we analyze the computational complexity with the base case, without loss of generality. When using Algorithm 1 to solve the base case RSCOPF model, the number of binary variables in MP (19) is $N B \times N T$, while the number of constraints are dependent on the number of the worst-case realizations obtained from the SP model (20). To solve the SP with the equivalent MILP model (see Appendix), the number of binary variables is $(N W+N L) \times N T \times 2$, the number of continuous variables is $(N W+N L) \times N T \times 4$.

Despite that the number of binary variables is large, we want to remark that the computational time to solve the proposed RSC-OPF model depends on the extent of operational inflexibility. For example, if the power system is flexible (without reluctant load shedding or wind curtailment), the binary variables associated with both MP and SP should be zero. Therefore, the MILP solve will obtain the solution with zero MILP gap by solving the corresponding LP relaxation models. The state-of-the-art MILP solver is also able to remove redundant variables and constraints with heuristics in the pre-process stage. Based on our simulation results, if the operational inflexibility is not significant (which is true for most realistic cases) and the number of insufficient flexibility events is small, the MILP solver is able to obtain the integer solution within reasonable computational time.

\section{CAse Studies}

In this section, comprehensive case studies are presented to demonstrate the applications of the proposed model using the IEEE 14-bus system and a realistic Chinese 157-bus system. The C\&CG algorithm and the modified BD are implemented on MATLAB with YALMIP. The MILP solver is CPLEX V.12.5. The MILP gap for the MILP solver is set to be $0.1 \%$. The termination tolerance of the C\&CG algorithm is $1 \%$. The case studies are performed on a personal computer with an 8-core $3.4 \mathrm{GHz}$ i7 processor and $6 \mathrm{~GB}$ RAM.

\section{A. Wind and Load Profiles}

The daily load and wind profiles in Fig.2 are taken from EirGrid system data on Nov. $21^{\text {st }} 2015$ (15-minute intervals) [47]. The actual total load demand is normalized by $4000 \mathrm{MW}$ to obtain the load profile curve, and the predicted total wind output is normalized by $840 \mathrm{MW}$ to obtain the wind profile curve. Based on the EirGrid data, the maximum wind forecast error on that day is $\pm 45 \%$.

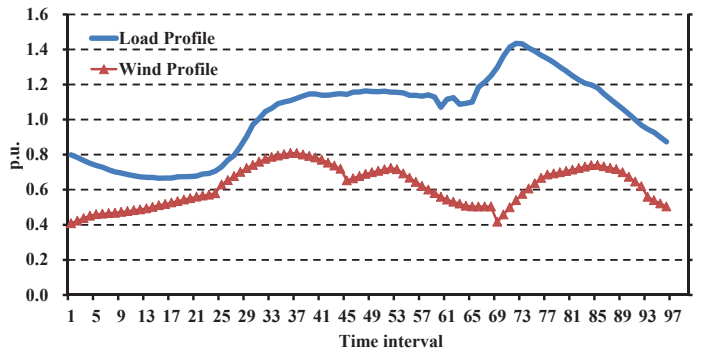

Fig. 2. Load and wind profiles

\section{B. IEEE 14-bus System Case}

\section{1) Computational settings:}

The IEEE 14-bus system data are taken from [48] and normalized by 100 MVA. The total load demand is $259 \mathrm{MW}$. The generators on bus 1, 2, 3, and 6 are dispatchable. The wind generator is located on bus 8 . The characteristics of generators are listed in Table I. Let $\tau^{\max }=15 \mathrm{~min}$. For ease of discussion, let $\Delta P_{i}^{\mathrm{g}, \mathrm{up}}=\Delta P_{i}^{\mathrm{re}, \mathrm{max}}=-\Delta P_{i}^{\mathrm{g}, \mathrm{dn}}=R U_{i} \times 15 \mathrm{~min}$. The bi-directional transmission limit of each branch is 150 MW. To investigate the impact of transmission constraints on the wind penetration level, the branch between bus 7 and bus 8 is replaced by two branches (denoted as (a) and (b) respectively), with a $75 \mathrm{MW}$ transmission limit each. Set the probabilities of contingencies as $p_{0}=1, p_{c}=0.02, \forall c>0$. The system topology is shown in Fig. 3. For a given forecast error $\delta$, the uncertainty sets associated with these two curves are spanned by $[1-\delta, 1+\delta]$.

TABLE I

GENERATOR CHARACTERISTICS OF IEEE 14-BUS SYSTEM

\begin{tabular}{cccc}
\hline Bus & $P \max (\mathrm{MW})$ & $\operatorname{Pmin}(\mathrm{MW})$ & $R U_{i}(\mathrm{MW} / 15 \mathrm{~min})$ \\
\hline 1 & 332.4 & 50 & 47.06 \\
2 & 150 & 0 & 25 \\
3 & 150 & 0 & 25 \\
6 & 150 & 0 & 25 \\
8 & 100 & N.A. & N.A. \\
\hline
\end{tabular}

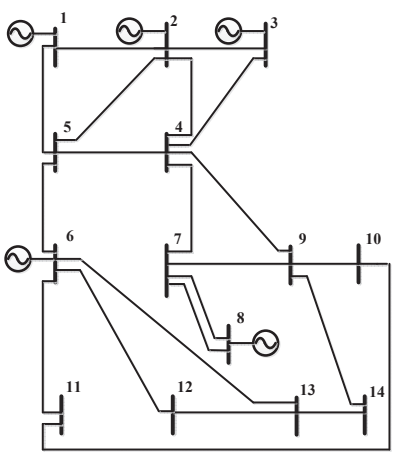

Fig. 3. IEEE 14-bus system

For this system, two cases are presented to demonstrate the applications of the proposed model. The first case visualizes the flexibility region and identifies the critical transmission paths, while the second case investigates the impact of ramping capacity on intra-hour operational flexibility. We define the wind penetration level $\beta$ to scale the wind profile in Fig. 2 .

2) Case 1: Flexibility region and critical transmission path$s:$

Let $\beta=1.2$ and the wind forecast error be $30 \%$. The load demand on each bus is scaled by the load profile in Fig. 2, with the forecast error set to $3 \%$. This case consists of a total of 12 buses with uncertain power injection/demand, including 1 wind generator bus plus 11 load buses. The flexibility assessment is conducted considering all N-1 contingencies. Two contingencies are used as examples to demonstrate the spatial-temporal relationship of insufficient flexibility and the sensitivity analysis. Considering the outage on branch 1-2, a 
14.09 MW shortage in generation occurs on bus 2 at time interval 41 . The sensitivity of the top 5 binding constraints are listed in Table II, which indicates an insufficiency in spinning reserve and contingency reserve. Considering the outage on one of the branches of 7-8, which is the most severe contingency, 79 insufficient flexibility events occur on bus 8 . The time intervals and the amount of wind curtailment associated with these events are depicted in Fig. 4. System operators can estimate the amount of wind curtailment versus time during system operation in the worst-case realization of wind output. The sensitivities of the top 5 binding constraints are listed in Table III, showing the insufficiency of transmission capacity.

TABLE II

BINDING CONSTRAINTS OF CASE 1: OUTAGE ON BRANCH 1-2

\begin{tabular}{cccc}
\hline Time Interval & Component & Constraint & Sensitivity \\
\hline 41 & Gen. on Bus 3 & (6b) Upper Bound & 2.00 (p.u./hr) \\
41 & Gen. on Bus 6 & (6b) Upper Bound & 2.00 (p.u./hr) \\
41 & Gen. on Bus 1 & (6b) Upper Bound & 1.88 (p.u./hr) \\
41 & Gen. on Bus 3 & (9) Upper Bound & 1.00 (p.u./p.u.) \\
41 & Gen. on Bus 2 & (6b) Upper Bound & 1.00 (p.u./hr) \\
\hline
\end{tabular}

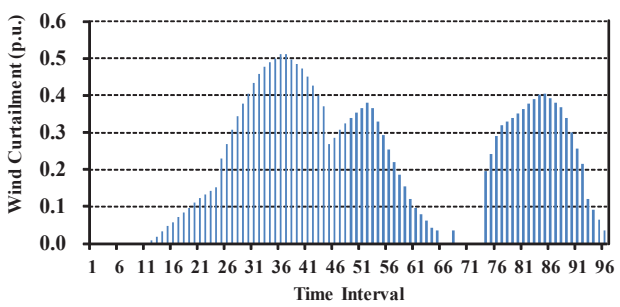

Fig. 4. Wind curtailment on bus 8 due to outage on branch 7-8 (a)

TABLE III

BINDING CONSTRAINTS OF CASE 1: OUTAGE ON ONE BRANCH OF 7-8 (A)

\begin{tabular}{cccc}
\hline Time Interval & Component & Constraint & Sensitivity \\
\hline 34 & Branch 7-8 & (5) Lower Bound & 1.00 (p.u./p.u.) \\
35 & Branch 7-8 & (5) Lower Bound & 1.00 (p.u./p.u.) \\
36 & Branch 7-8 & (5) Lower Bound & 1.00 (p.u./p.u.) \\
37 & Branch 7-8 & (5) Lower Bound & 1.00 (p.u./p.u.) \\
38 & Branch 7-8 & (5) Lower Bound & 1.00 (p.u./p.u.) \\
\hline
\end{tabular}

For the outage on one of the branches of 7-8, as indicated in Fig. 4, the FIF is 79/96, the MIF is $2099.98 \mathrm{MW}$, and the IIF is $26.58 \mathrm{MW} /$ event.

As for the computational complexity of the RSC-OPF model for this case, for each contingency (the base case is regarded as contingency 0$)$, MP includes $1152(12 \times 96)$ binary variables, SP includes $2304(12 \times 2 \times 96)$ binary variables. Considering total 22 contingencies, the $\mathrm{BD}$ process converges by 5 iterations. The total CPU time is $1648 \mathrm{~s}$. The average $\mathrm{CPU}$ time to solve the base case sub-model is $2 \mathrm{~s}$. The average CPU time to solve each $\mathrm{N}-1$ contingency sub-model is $12 \mathrm{~s}$. The average CPU time to generate the Benders cut for each $\mathrm{N}-1$ contingency is $2 \mathrm{~s}$. The total CPU time can be reduced if the contingency sub-models are solved in parallel. By contrast, without BD process, the total number of binary variables will be increased by 22 times into one single model. The CPU time to solve this large-scale $\mathrm{RO}$ model is $8.1 \mathrm{~h}$. The BD process reduces the computational complexity substantially with the relative difference of $0.93 \%$ in objective value.

To investigate the interplay between the wind penetration level $\beta$ and the forecast error on the operational flexibility, we perturb $\beta$ from 0.8 to 1.2 by increments of 0.05 , and perturb the wind forecast error from $5 \%$ to $30 \%$ by increments of $5 \%$. The flexibility assessment is conducted with 54 combinations of $\beta$ and wind forecast error. The contour of the MIF (in p.u.) is depicted in Fig. 5, from which it can be seen that the acceptable forecast error drops as the wind penetration level increases. The wind penetration level for this system has an upper bound due to the limitation of the transmission capacity. The contour line with the smallest MIF value (i.e., 0.004 p.u. in Fig. 5 (top)) can be regarded as the boundary of the flexibility region spanned by the forecast error and the wind penetration level.

We then calculated the average MIF associated with each $\mathrm{N}-1$ contingencies over the 54 combinations of the forecast error and wind penetration level. The most important five branches are identified in Table IV. The first two branches imply that a transmission expansion may be needed to improve the wind penetration level. The rest of the branches imply that the spinning reserve and contingency reserve are slightly insufficient in the presence of the corresponding contingency, since all the transmission limit constraints are not binding.
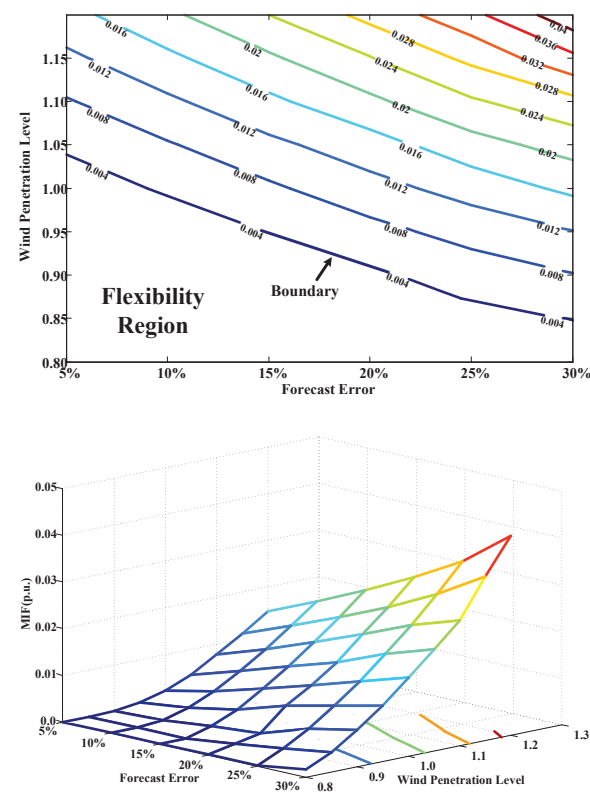

Fig. 5. Flexibility region (p.u.) (top) and contour of MIF (p.u.) (bottom) of Case 1

TABLE IV

IMPORTANCE RANKING OF BRANCHES

\begin{tabular}{cc}
\hline Branch & Average MIF (MW) \\
\hline $7-8(\mathrm{a})$ & 11.46 \\
$7-8(\mathrm{~b})$ & 11.46 \\
$2-3$ & 0.030 \\
$6-13$ & 0.026 \\
$3-4$ & 0.025 \\
\hline
\end{tabular}


3) Case 2: Ramping capacity assessment:

In this case, the proposed model is used to assess the impact of ramping capacity on intra-hour operational flexibility, identifying the worst-case scenario of wind output and ramping events. Furthermore, the spatial and temporal characteristics of power imbalance due to lack of ramping capacity, if any, will be quantified using the proposed model.

For this case, we set $\beta=1.45$, the load demand to be deterministic, and set the wind forecast error to $25 \%$. Additionally, the ramping rates of the dispatchable generators are reduced to $50 \%$ of the value in the $4^{\text {th }}$ column of Table I. The assessment is performed for the base case. The minimum wind output, the maximum wind output, and the worst-case realization of wind output obtained by RSC-OOPF, are depicted in Fig. 6. For this worst-case realization, the most severe wind ramping down event takes place on time interval 29, when the load demand is sharply increasing. In this worst-case realization of wind profile at time interval 29, the wind output is 131 MW. At time interval 30, the wind output drops to $80 \mathrm{MW}$. Meanwhile, the load ramps from 234 MW to 251.7 MW. The total net load increases by $68 \mathrm{MW}$ within 15 mins. By contrast, the total ramping capacities of all dispatchable generators sum up to $61 \mathrm{MW}$. A shortage in ramping capacity of $7 \mathrm{MW}$ takes place at time interval 29.

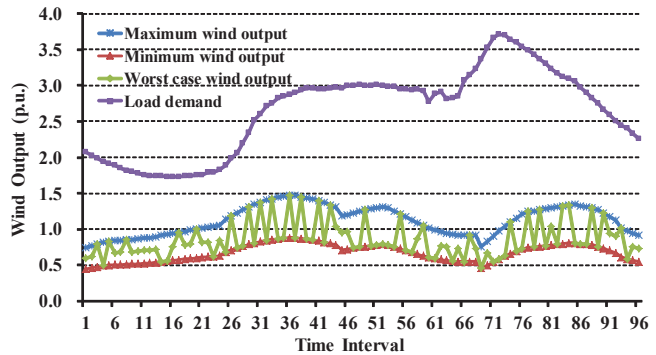

Fig. 6. Worst-case realization of wind output obtained by RSC-OPF

A total of 5 power imbalance events occur in this time horizon, as shown in Fig. 7. The MIF associated with this worst-case realization is $13.60 \mathrm{MW}$. System operators can derive the load shedding and wind curtailment on the identified buses at appropriate time interval to maintain generation-load balancing. The top 5 binding constraints and the corresponding sensitivities are listed in Table $\mathrm{V}$, indicating that an insufficiency in ramping capacity results in insufficient flexibility.

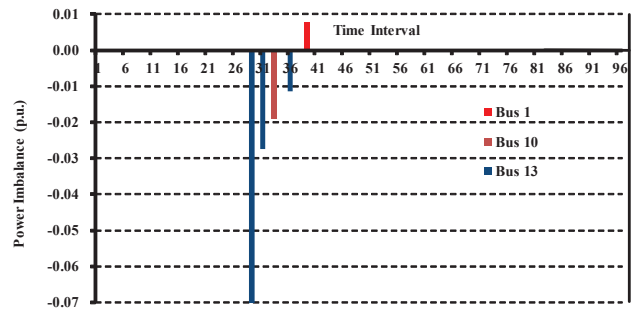

Fig. 7. Power imbalance events due to insufficient flexibility
TABLE $\mathrm{V}$

BINDING CONSTRAINTS OF CASE 2

\begin{tabular}{cccc}
\hline Time & Component & Constraint & Sensitivity \\
\hline 29 & Gens. on bus $1,2,3,6$ & (7) Upper Bound & 1.00 (p.u./p.u.) \\
31 & Gens. on bus $1,2,3,6$ & (7) Upper Bound & 1.00 (p.u./p.u.) \\
33 & Gens. on bus $1,2,3,6$ & (7) Upper Bound & 1.00 (p.u./p.u.) \\
36 & Gens. on bus $1,2,3,6$ & (7) Upper Bound & 1.00 (p.u./p.u.) \\
38 & Gens. on bus $1,2,3,6$ & (7) Lower Bound & 1.00 (p.u./p.u.) \\
\hline
\end{tabular}

To validate the aforementioned worst-case realization wind output, we apply Monte Carlo Simulation to generate 2000 wind output curves using the uniform distribution between maximum and minimum wind output, and subsequently solve RSC-OPF with these curves. The worst-case realization of wind output among these 2000 curves is depicted as in Fig. 8. The MIF associated with this worst-case realization of wind output is $10.20 \mathrm{MW}$ (less than 13.60 MW identified by RSCOPF), which could be over-optimistic.

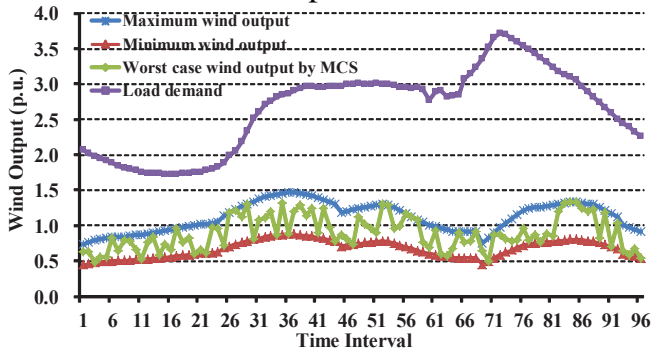

Fig. 8. Worst-case realization of wind output obtained by Monte Carlo simulation

\section{Chinese 157-bus System Case}

The 157-bus system [49] in Gansu, China is part of Chinese northwest grid. The characteristics of this system is listed in Table VI. The total capacity of the thermal plants is 21951 MW, the total capacity of the hydro plants is $7938 \mathrm{MW}$, the total load demand is $22903 \mathrm{MW}$, and the total wind output is $6722 \mathrm{MW}$. The minimum output of the hydro plants is $30 \%$, and the minimum technical output of the thermal plants is $55 \%$. The load and wind output are scaled by the curves in Fig. 2. The forecast error of wind output is set to $20 \%$.

The flexibility assessment is conducted for the base case. By solving the RSC-OPF model, the aggregate power imbalances are depicted in Fig. 9. During load valley periods (i.e., time intervals between 10 and 25), wind curtailment occurs due to the minimum technical output of thermal plants, whereas during load peak periods (i.e., time intervals between 70 and 75), load shedding occurs due to the spinning reserve constraints. The binding constraints are listed in Table VII, showing the insufficient spinning reserves are the major cause of insufficient flexibility on the base case.

TABLE VI

System Characteristics of THE Chinese 157-Bus System

\begin{tabular}{cc|cc}
\hline Component & Number & Component & Number \\
\hline Lines & 228 & Thermal Plants & 29 \\
Transformers & 30 & Hydro Plants & 40 \\
Buses & 157 & Wind Farms & 29 \\
\hline
\end{tabular}




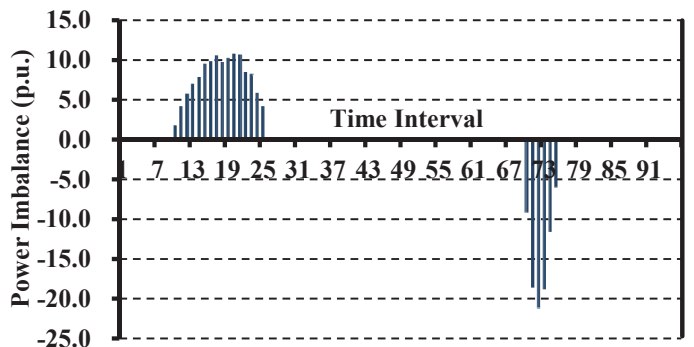

Fig. 9. Aggregate power imbalance of the base case Chinese 157-bus System

TABLE VII

BINDING CONSTRAINTS OF BASE CASE OF 157-BUS SYSTEM

\begin{tabular}{cccc}
\hline Time & Component & Constraint & Sensitivity \\
\hline 70 & Gens. on bus 6,7,43,46 & (6a) Upper Bound & 1.00 (p.u./p.u.) \\
71 & Gens. on bus 6,7,43,46 & (6a) Upper Bound & 1.00 (p.u./p.u.) \\
72 & Gens. on bus 6,7,43,46 & (6a) Upper Bound & 1.00 (p.u./p.u.) \\
73 & Gens. on bus 6,7,43,46 & (6a) Upper Bound & 1.00 (p.u./p.u.) \\
74 & Gens. on bus 6,7,43,46 & (6a) Upper Bound & 1.00 (p.u./p.u.) \\
\hline
\end{tabular}

The comparisons between hourly and intra-hour assessments are listed in Table. VIII, which shows that hourly operational assessment may not cover all insufficient flexibility events. The spinning reserve is critical, otherwise the flexibility assessment would be over-optimistic. This table also presents the computational time to demonstrate the performance of the proposed method. For the 96 time interval flexibility assessment considering the reserve constraint (6), the C\&CG algorithm takes 2 iterations to obtain the final solution. The MP model is solved twice, with the average computational time as $10.2 \mathrm{~s}$. The SP model is solved once to identify the worst-case realization of wind output, with the computational time as $240 \mathrm{~s}$. Considering 30 different $\mathrm{N}-1$ contingencies to trip one of the parallel transmission lines, the BD process converges in 4 iterations. Each iteration needs to solve the base case sub-model for once, and the contingency sub-models for 30 times. The total computational time is $12.3 \mathrm{~h}$. If the contingency sub-models are solved in a parallel manner, the computation time will be reduced considerably. Without $\mathrm{BD}$ process, the RSC-OPF model for this 157-bus system considering various contingencies is intractable.

TABLE VIII

COMPUTATIONAL RESULTS UNDER DIFFERENT CONFIGURATIONS

\begin{tabular}{cccccc}
\hline Constraints & $\begin{array}{c}\text { Time } \\
\text { Resolution }\end{array}$ & FIF & $\begin{array}{c}\text { MIF } \\
\text { (p.u.) }\end{array}$ & $\begin{array}{c}\text { IIF } \\
\text { (p.u./event) }\end{array}$ & $\begin{array}{c}\text { CPU } \\
\text { Time (s) }\end{array}$ \\
\hline with (6) & $60 \mathrm{~min}$ & $5 / 24$ & 52.89 & 10.58 & 62 \\
without (6) & $15 \mathrm{~min}$ & $16 / 96$ & 122.72 & 7.67 & 184 \\
with (6) & $15 \mathrm{~min}$ & $22 / 96$ & 207.50 & 9.43 & 276 \\
\hline
\end{tabular}

From the system operator's viewpoint, it is important to identify the least number of load shedding actions. Take the assessment on time interval 72 as an example. The systemspecific parameter $\Delta P_{i}^{\mathrm{im}, \max }$ of each load bus is varied from $10 \%$ to $30 \%$ of its predicted output. The minimum number of buses with load shedding for each value of $\Delta P_{i}^{\mathrm{im}, \max }$ are depicted in Fig. 10. A tradeoff can be achieved between the amount of load shedding and the number of affected buses. By contrast, if the number of buses with power imbalance is not minimized in the $1^{\text {st }}$ stage (as in (18)), the power balance will take place in 92 buses, i.e., all load and generation buses.

The flexibility region of this system is depicted in Fig. 11, showing insufficient flexibility occurs as wind output decreases, as a result of spinning reserve constraints. In other words, the wind penetration level for this system has a lower bound due to the limitation of spinning reserve.

It is seen from the simulation results that the lower bound of wind penetration level is primarily determined by the spinning reserve constraints (binding at time intervals between 70 and 75). During this period, the wind output is decreasing whereas the output of conventional units cannot increase since the reserve capacities are limited. Quick-start units could be applied to mitigate the insufficient flexibility during this period. The upper bound of wind penetration level is restricted by the minimum technical output of thermal units, as the wind curtailment occurs at time intervals between 10 and 15 . The wind penetration level could be improved during that period by applying pump storage units to store the excessive generation.

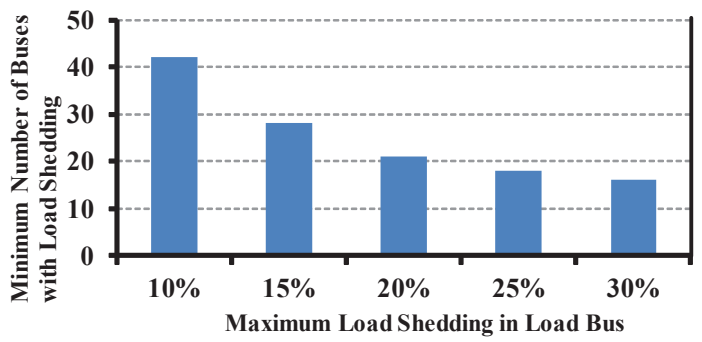

Fig. 10. Minimum number of buses with load shedding
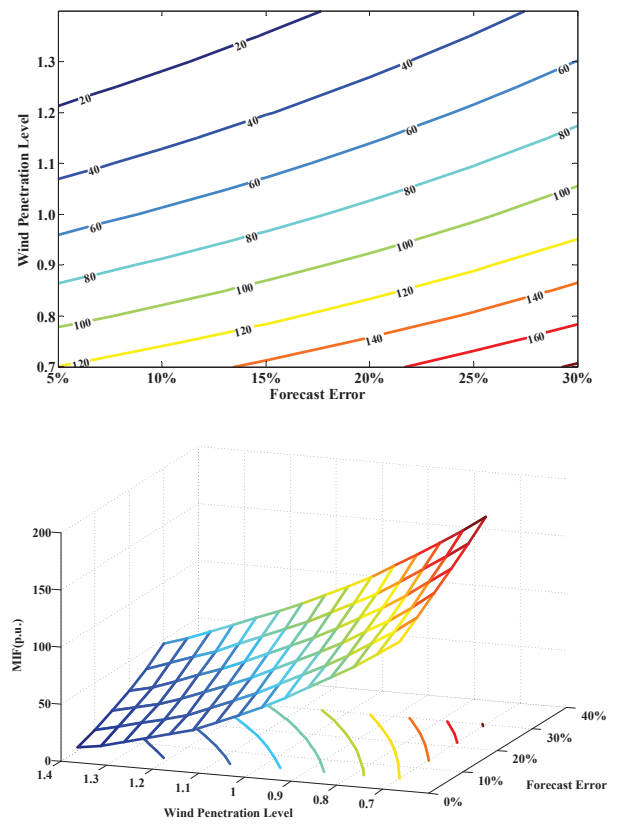

Fig. 11. Flexibility region (top) and contour of MIF (p.u.) (bottom) of Chinese 157-bus system on the base case

\section{Discussions}

The above case studies indicate that spinning reserve and contingency reserve are critical for hedging the risk arising 
from the uncertainty of both RES and unexpected transmission/generation outages over intra-hour time scales. While the case studies are performed for 24 hours, the proposed method can also be applied in a rolling framework to conduct intrahour flexibility assessment for 4-6 hours. With the continuous update on the wind prediction, the flexibility region will help system operators aware the impact of RES output on the operational flexibility. Emergency measures can be prepared to safeguard the worst-case realization of RES output.

The proposed model, establishing basic results, can be generalized to include the uncertainty budget, penalty/cost terms on the load shedding and wind curtailment.

The flexibility regions demonstrated in the case studies are obtained by assuming the RES penetration levels and the associated forecast errors are consistent throughout the power grid. However, the concept of flexibility region can be applied to study operational flexibility with diverse RES power injections, which lead to a high-dimensional flexibility region. This high-dimensional flexibility region can be visualized with projection onto some particular bus.

The flexibility region is different from the power flow feasibility region (which is generally determined by the power injections, power flow equations and operational constraints) in that the power flow balance in the RSC-OPF model is regarded as a soft constraint due to the introduced slack variables in (12). We apply the flexibility region to characterize the operational flexibility in terms of the amount of power imbalance. In other words, this region is able to coherently characterize the power system from feasible to infeasible in the sense of DC power flow.

\section{Conclusion}

It is critical to assess the operational flexibility to accommodate a high penetration of RES with existing resources.

To investigate intra-hour operational flexibility, an RO model is constructed to quantify the insufficient flexibility. The proposed method provides system operators with the following key results: 1) the minimum and/or maximum range of RES penetration level and the forecast error (i.e., flexibility region); 2) quantitative metrics if the RES output is beyond the flexibility region; 3) a worst-case combination of the RES output profile and the unexpected outage, which together incur insufficient flexibility over a studied time horizon; 4) emergency measures on a limited number of affected buses if insufficient flexibility occurs.

Future work would investigate how the insufficient flexibility metrics provide incentives for new investments to achieve equilibrium of operational flexibility.

\section{APPENDIX \\ REFORMULATION OF SP INTO MILP}

In this Appendix, the reformulation of the subproblem (SP) of RO into a MILP model is briefly introduced. Note that the SP in (20) should yield a new worst-case realization of $\tilde{d}$, whether the optimal solution of the $1^{\text {st }}$ stage variable $\boldsymbol{x}^{*}$ leads to a feasible SP in (20) or not. To this end, the SP in (20) is converted into an infeasibility detection model given by

$$
\begin{array}{ll} 
& Q\left(\boldsymbol{x}^{*}\right)=\max _{\tilde{\boldsymbol{d}}} \min _{\boldsymbol{y}_{+}, \boldsymbol{y}_{-}, \boldsymbol{s}, \boldsymbol{s}_{1}, \boldsymbol{s}_{2}} \boldsymbol{b}^{T}\left(\boldsymbol{y}_{+}-\boldsymbol{y}_{-}\right)+\boldsymbol{\rho}^{T}\left(\boldsymbol{s}_{1}+\boldsymbol{s}_{2}\right) \\
\text { s.t. } & \boldsymbol{N}\left(\boldsymbol{y}_{+}-\boldsymbol{y}_{-}\right)+\left(\boldsymbol{s}_{1}-\boldsymbol{s}_{2}\right)=\tilde{\boldsymbol{d}} \\
& \boldsymbol{M}\left(\boldsymbol{y}_{+}-\boldsymbol{y}_{-}\right)-\boldsymbol{s}=\boldsymbol{m} \\
& \boldsymbol{y}_{+} \geq 0, \boldsymbol{y}_{-} \geq 0, \boldsymbol{s} \geq 0, \boldsymbol{s}_{1} \geq 0, \boldsymbol{s}_{2} \geq 0 \\
& \tilde{\boldsymbol{d}} \in\left[\boldsymbol{d}_{\min }, \boldsymbol{d}_{\max }\right]
\end{array}
$$

where $\rho$ is a vector of a sufficiently large number (e.g., 1E4) with the compatible size. If $\boldsymbol{\rho}^{T}\left(\boldsymbol{s}_{1}+\boldsymbol{s}_{2}\right) \rightarrow 0$ by solving (26), a worst-case realization of $\tilde{\boldsymbol{d}}$, which leads to a feasible SP in (20), is generated. Otherwise, a worst-case realization of $\tilde{\boldsymbol{d}}$ leading to an infeasible SP in (20) is identified. In either case, this new worst-case realization will be fed into the MP to enforce a change in the $1^{\text {st }}$ stage variables due to the feasibility cut $(18 \mathrm{~g})$.

Note that the inner minimization problem in (26), including the objective function and (26b)-(26d), is a standard LP model. By merging the dual of the inner minimization problem, (26) can be recast into a non-convex bilinear programming model as below.

$$
\begin{array}{ll} 
& Q\left(\boldsymbol{x}^{*}\right)=\max _{\tilde{\boldsymbol{d}}, \boldsymbol{w}_{1}, \boldsymbol{w}_{2}} \tilde{\boldsymbol{d}}^{T} \boldsymbol{w}_{1}+\boldsymbol{m}^{T} \boldsymbol{w}_{2} \\
\text { s.t. } & \boldsymbol{N}^{T} \boldsymbol{w}_{1}+\boldsymbol{M}^{T} \boldsymbol{w}_{2}=\boldsymbol{b} \\
& \boldsymbol{w}_{2} \geq 0 \\
& -\boldsymbol{\rho} \leq \boldsymbol{w}_{1} \leq \boldsymbol{\rho} \\
& \boldsymbol{d}_{\min } \leq \tilde{\boldsymbol{d}} \leq \boldsymbol{d}_{\max }
\end{array}
$$

For the polyhedral uncertainty set in (27e), we only need to consider its vertices to identify a worst-case realization (see Theorem 1 in [50]). Therefore, (27e) can be reformulated using a pair of binary variables $Z_{+}$and $Z_{-}$, as follows,

$$
\tilde{d}=\bar{d}+\left(Z_{+}-Z_{-}\right) \Delta d
$$

where $\Delta \boldsymbol{d}$ and $\overline{\boldsymbol{d}}$ are the deviation and average value of random variables, respectively, given by

$$
\begin{aligned}
\Delta \boldsymbol{d} & =\frac{\boldsymbol{d}_{\mathrm{max}}-\boldsymbol{d}_{\mathrm{min}}}{2} \\
\overline{\boldsymbol{d}} & =\frac{\boldsymbol{d}_{\max }+\boldsymbol{d}_{\mathrm{min}}}{2}
\end{aligned}
$$

Plug (28) and (29) into (27), a non-convex bilinear model with the production of binary variables and continuous variables in the objective function is given by

$$
\begin{aligned}
Q\left(\boldsymbol{x}^{*}\right)= & \max _{\boldsymbol{Z}_{+}, \boldsymbol{Z}_{-}, \boldsymbol{w}_{1}, \boldsymbol{w}_{2}} \overline{\boldsymbol{d}}^{T} \boldsymbol{w}_{1}+\boldsymbol{m}^{T} \boldsymbol{w}_{2} \\
& +\left(\operatorname{diag}\left(\boldsymbol{Z}_{+}\right) \boldsymbol{w}_{1}-\operatorname{diag}\left(\boldsymbol{Z}_{-}\right) \boldsymbol{w}_{1}\right)^{T} \Delta \boldsymbol{d} \\
\text { s.t. } & (27 \mathrm{~b}),(27 \mathrm{c}),(27 \mathrm{~d}) \\
& \boldsymbol{Z}_{+}+\boldsymbol{Z}_{-} \leq 1
\end{aligned}
$$

where $\operatorname{diag}(\bullet)$ denote the transformation of an array into the corresponding diagonal matrix. We introduce two variables $\boldsymbol{k}_{1}$ 
and $\boldsymbol{k}_{2}$ to represent the production of binary variables and continuous variables, given by

$$
\begin{aligned}
& \boldsymbol{k}_{1}=\operatorname{diag}\left(\boldsymbol{Z}_{+}\right) \boldsymbol{w}_{1} \\
& \boldsymbol{k}_{2}=\operatorname{diag}\left(\boldsymbol{Z}_{-}\right) \boldsymbol{w}_{1}
\end{aligned}
$$

Subsequently, the following McCormick cuts are applied to exactly convert the production of binary variables and continuous variables into linear inequalities, as follows.

$$
\begin{aligned}
& -\operatorname{diag}\left(\boldsymbol{Z}_{+}\right) \boldsymbol{\rho} \leq \boldsymbol{k}_{1} \leq \operatorname{diag}\left(\boldsymbol{Z}_{+}\right) \boldsymbol{\rho} \\
& -\operatorname{diag}\left(1-\boldsymbol{Z}_{+}\right) \boldsymbol{\rho} \leq \boldsymbol{k}_{1}-\boldsymbol{w}_{1} \leq \operatorname{diag}\left(1-\boldsymbol{Z}_{+}\right) \boldsymbol{\rho} \\
& -\operatorname{diag}\left(\boldsymbol{Z}_{-}\right) \boldsymbol{\rho} \leq \boldsymbol{k}_{2} \leq \operatorname{diag}\left(\boldsymbol{Z}_{-}\right) \boldsymbol{\rho} \\
& -\operatorname{diag}\left(1-\boldsymbol{Z}_{-}\right) \boldsymbol{\rho} \leq \boldsymbol{k}_{2}-\boldsymbol{w}_{2} \leq \operatorname{diag}\left(1-\boldsymbol{Z}_{-}\right) \boldsymbol{\rho}
\end{aligned}
$$

Plug (31) and (32) into (30), a MILP model is established to solve (20) as follows.

$$
\begin{aligned}
Q\left(\boldsymbol{x}^{*}\right) & =\max _{\boldsymbol{Z}_{+}, \boldsymbol{Z}_{-}, \boldsymbol{w}_{1}, \boldsymbol{w}_{2}, \boldsymbol{k}_{1}, \boldsymbol{k}_{2}} \overline{\boldsymbol{d}}^{T} \boldsymbol{w}_{1}+\boldsymbol{m}^{T} \boldsymbol{w}_{2} \\
& +\left(\boldsymbol{k}_{1}-\boldsymbol{k}_{2}\right)^{T} \Delta \boldsymbol{d} \\
\text { s.t. } & (27 \mathrm{~b}),(27 \mathrm{c}),(27 \mathrm{~d}),(32) \\
& \boldsymbol{Z}_{+}+\boldsymbol{Z}_{-} \leq 1
\end{aligned}
$$

It is seen from above that, for each random variable, a pair of binary variables and 4 continuous variables will be introduced in the MILP formulation of SP.

To account for uncertainty budget, one can include additional restriction on the number of 1 element in the array of $\boldsymbol{Z}_{+}$ and $\boldsymbol{Z}_{-}$. Since the optimal solution of (33) lies in the vertices of the polyhedral uncertainty set, the optimal solution will be obtained within a limited number of iterations, see Proposition 1 in [42].

\section{ACKNOWLEDGMENT}

We would like to thank Prof. Felix F. Wu with University of California, Berkeley, and Prof. Chen-Ching Liu with Washington State University, for their valuable suggestions. We would also like to thank the anonymous reviewers for their insightful and very constructive comments that have substantially helped to improve both the technical content and the presentation of this paper.

\section{REFERENCES}

[1] E. Ela and M. O'Malley, "Studying the variability and uncertainty impacts of variable generation at multiple timescales," IEEE Trans. Power Syst., vol. 27, no. 3, pp. 1324-1333, Aug. 2012.

[2] North American Electric Reliability Corporation (NERC), "Flexibility requirements and potential metrics for variable generation: implications for system planning studies," http://www.nerc.com/files/ IVGTFTask14Final.pdf, 2010, [Online; accessed 9-November-2015].

[3] H. Holttinen, A. Tuohy, M. Milligan, E. Lannoye, V. Silva, S. Muller and L. Soder, "The flexibility workout: Managing variable resources and assessing the need for power system modification," IEEE Power Energy Mag., vol. 11, no. 6, pp. 53-62, Nov. 2013.

[4] European Wind Energy Association, Wind Energy - The Facts: A Guide to the Technology, Economics and Future of Wind Power, 1st ed. Routledge, March 2009.

[5] M. Bucher, S. Chatzivasileiadis, and G. Andersson, "Managing flexibility in multi-area power systems," IEEE Trans. Power Syst., vol. PP, no. 99 , pp. 1-9, 2015.

[6] X. Chen, C. Kang, M. O’Malley, Q. Xia, J. Bai, C. Liu, R. Sun, W. Wang, and $\mathrm{H}$. Li, "Increasing the flexibility of combined heat and power for wind power integration in China: Modeling and implications," IEEE Trans. Power Syst., vol. 30, no. 4, pp. 1848-1857, Jul. 2015.
[7] A. Spisto, T. Mobius, and S. Quoilin, "Demand for flexibility in the power system under different shares of renewable generation," in Proc. 2015 12th International Conference on the European Energy Market (EEM), May 2015, pp. 1-5.

[8] C. O'Dwyer and D. Flynn, "Using energy storage to manage high net load variability at sub-hourly time-scales," IEEE Trans. Power Syst., vol. 30, no. 4, pp. 2139-2148, Jul. 2015.

[9] A. Muzhikyan, A. Farid, and K. Youcef-Toumi, "Variable energy resource induced power system imbalances: Mitigation by increased system flexibility, spinning reserves and regulation," in Proc. 2013 1st IEEE Conference on Technologies for Sustainability (SusTech), Aug. 2013, pp. 15-22.

[10] B. Zhang and M. Kezunovic, "Impact on power system flexibility by electric vehicle participation in ramp market," IEEE Trans. Smart Grid, vol. PP, no. 99, pp. 1-1, 2015.

[11] A. Rosso, J. Ma, D. Kirschen, and L. Ochoa, "Assessing the contribution of demand side management to power system flexibility," in Proc. 2011 50th IEEE Conference on Decision and Control and European Control Conference (CDC-ECC), Dec. 2011, pp. 4361-4365.

[12] H. Wu, M. Shahidehpour, A. Alabdulwahab, and A. Abusorrah, "Thermal generation flexibility with ramping costs and hourly demand response in stochastic security-constrained scheduling of variable energy sources," IEEE Trans. Power Syst., vol. 30, no. 6, pp. 2955-2964, Nov. 2015.

[13] C. Sahin, M. Shahidehpour, and I. Erkmen, "Allocation of hourly reserve versus demand response for security-constrained scheduling of stochastic wind energy," IEEE Trans. Sustain. Energy, vol. 4, no. 1, pp. 219-228, Jan. 2013

[14] H. Wu, M. Shahidehpour, and A. Al-Abdulwahab, "Hourly demand response in day-ahead scheduling for managing the variability of renewable energy," IET Gener. Transm. Distrib., vol. 7, no. 3, pp. 226-234. Mar. 2013.

[15] H. Wu, M. Shahidehpour, A. Alabdulwahab, and A. Abusorrah, "Thermal generation flexibility with ramping costs and hourly demand response in stochastic security-constrained scheduling of variable energy sources,” IEEE Trans. Power Syst., vol. 30, no. 6, pp. 2955-2964, Nov. 2015.

[16] N. G. Paterakis, O. Erdinc, A. G. Bakirtzis, and J. P. S. Catalo, "Load-following reserves procurement considering flexible demand-side resources under high wind power penetration," IEEE Trans. Power Syst., vol. 30, no. 3, 2015.

[17] C. E. Murillo-Snchez, R. D. Zimmerman, C. L. Anderson, and R. J. Thomas, "Secure planning and operations of systems with stochastic sources, energy storage, and active demand," IEEE Trans. Smart Grid, vol. 4, no. 4, pp. 2220-2229, Dec. 2013.

[18] N. G. Paterakis, O. Erdin, A. G. Bakirtzis, and J. P. S. Catalo, "Qualification and quantification of reserves in power systems under high wind generation penetration considering demand response," IEEE Trans. Sustain. Energy, vol. 6, no. 1, pp. 88-103, Jan. 2015.

[19] N. Maizi, V. Mazauric, E. Assoumou, and M. Drouineau, "Long-term planning and the sustainable power system: A focus on flexibility needs and network reliability," in Proc. 2009 IEEE/PES Power Systems Conference and Exposition (PSCE '09), Mar. 2009, pp. 1-6.

[20] E. Lannoye, D. Flynn, and M. O'Malley, "Transmission, variable generation, and power system flexibility," IEEE Trans. Power Syst., vol. 30, no. 1, pp. 57-66, Jan. 2015.

[21] F. Bouffard and M. Ortega-Vazquez, "The value of operational flexibility in power systems with significant wind power generation," in Proc. 2011 IEEE Power and Energy Society General Meeting, Jul. 2011, pp. 1-5.

[22] E. Lannoye, D. Flynn, and M. O'Malley, "Evaluation of power system flexibility," IEEE Trans. Power Syst., vol. 27, no. 2, pp. 922-931, May 2012.

[23] A. Thatte and L. Xie, "A metric and market construct of inter-temporal flexibility in time-coupled economic dispatch," IEEE Trans. Power Syst., vol. PP, no. 99, pp. 1-10, 2015.

[24] J. Ma, V. Silva, R. Belhomme, D. Kirschen, and L. Ochoa, "Evaluating and planning flexibility in sustainable power systems," IEEE Trans. Sustain. Energy, vol. 4, no. 1, pp. 200-209, Jan. 2013.

[25] K. Van Horn, "A network-inclusive, optimization-based approach to power system flexibility evaluation," in Proc. 2014 North American Power Symposium (NAPS), Sep. 2014, pp. 1-8.

[26] M. Bucher, S. Delikaraoglou, K. Heussen, P. Pinson, and G. Andersson, "On quantification of flexibility in power systems," in Proc. 2015 IEEE PowerTech Eindhoven, Jun. 2015, pp. 1-6.

[27] A. Ulbig and G. Andersson, "Analyzing operational flexibility of electric power systems," in Proc. 2014 Power Systems Computation Conference (PSCC), Aug. 2014, pp. 1-8. 
[28] J. Zhao, T. Zheng, and E. Litvinov, "A unified framework for defining and measuring flexibility in power system," IEEE Trans. Power Syst., vol. PP, no. 99, pp. 1-9, 2015.

[29] J. Zhao, T. Zheng and E. Litvinov, "Variable resource dispatch through do-not-exceed limit," IEEE Trans. Power Syst., vol. 30, no. 2, pp. 820828, Mar. 2015

[30] C. Wang, F. Liu, J. Wang, W. Wei, and S. Mei, "Risk-based admissibility assessment of wind generation integrated into a bulk power system," IEEE Trans. Sustain. Energy, vol. 7, no. 1, pp. 325-336, Jan. 2016.

[31] H. Nosair and F. Bouffard, "Reconstructing operating reserve: Flexibility for sustainable power systems," IEEE Trans. Sustain. Energy, vol. 6, no. 4, pp. 1624-1637, Oct. 2015.

[32] Y. Dvorkin, D. Kirschen, and M. Ortega-Vazquez, "Assessing flexibility requirements in power systems," IET Gener. Transm. Distrib., vol. 8 , no. 11 , pp. 1820-1830, 2014

[33] I. G. Marneris, P. N. Biskas, and E. A. Bakirtzis, "An integrated scheduling approach to underpin flexibility in European power systems," IEEE Trans. Sustain. Energy, vol. 7, no. 2, pp. 647-657, Apr. 2016.

[34] A. Ulbig and G. Andersson, "On operational flexibility in power systems," in Proc. 2012 IEEE Power and Energy Society General Meeting, Jul. 2012, pp. 1-8.

[35] H. Nosair and F. Bouffard, "Flexibility envelopes for power system operational planning," IEEE Trans. Sustain. Energy, vol. 6, no. 3, pp. 800-809, Jul. 2015

[36] W. A. Bukhsh, C. Zhang, and P. Pinson, "An integrated multiperiod OPF model with demand response and renewable generation uncertainty," IEEE Trans. Smart Grid, vol. 7, no. 3, pp. 1495-1503, May 2016.

[37] J. Wang, M. Shahidehpour, and Z. Li, "Security-constrained unit commitment with volatile wind power generation," IEEE Trans. Power Syst. vol. 23, no. 3, pp. 1319-1327, Aug. 2008.

[38] D. Bertsimas, E. Litvinov, X. A. Sun, J. Zhao, and T. Zheng, "Adaptive robust optimization for the security constrained unit commitment problem," IEEE Trans. Power Syst., vol. 28, no. 1, pp. 52-63, Feb. 2013.

[39] A. Street, F. Oliveira, and J. M. Arroyo, "Contingency-constrained unit commitment with $\mathrm{n}-\mathrm{k}$ security criterion: A robust optimization approach," IEEE Trans. Power Syst., vol. 26, no. 3, pp. 1581-1590, Aug. 2011.

[40] Y. Dvorkin, H. Pandi, M. A. Ortega-Vazquez, and D. S. Kirschen, "A hybrid stochastic/interval approach to transmission-constrained unit commitment," IEEE Trans. Power Syst., vol. 30, no. 2, pp. 621-631, Mar. 2015.

[41] Y. Fu, Z. Li, and L. Wu, "Modeling and solution of the largescale security-constrained unit commitment," IEEE Trans. Power Syst. vol. 28, no. 4, pp. 3524-3533, Nov. 2013.

[42] B. Zeng and L. Zhao, "Solving two-stage robust optimization problems using a column-and-constraint generation method," Operations Research Letters, vol. 41, no. 5, pp. 457 - 461, 2013.

[43] B. Hu and L. Wu, "Robust SCUC considering continuous/discrete uncertainties and quick-start units: A two-stage robust optimization with mixed-integer recourse," IEEE Trans. Power Syst., vol. PP, no. 99, pp. $1-13,2015$

[44] Z. Qin, Y. Hou, C.-C. Liu, S. Liu, and W. Sun, "Coordinating generation and load pickup during load restoration with discrete load increments and reserve constraints," IET Gener. Transm. Distrib., vol. 9, no. 15, pp. 2437-2446, Nov. 2015.

[45] Y. Fu, M. Shahidehpour, and Z. Li, "Ac contingency dispatch based on security-constrained unit commitment," IEEE Trans. Power Syst., vol. 21, no. 2, pp. 897-908, May 2006.

[46] Y. Wen, C. Guo, H. Pandi, and D. S. Kirschen, "Enhanced securityconstrained unit commitment with emerging utility-scale energy storage," IEEE Trans. Power Syst., vol. 31, no. 1, pp. 652-662, Jan. 2016.

[47] "Eirgrid system information," http://www.eirgridgroup.com/ how-the-grid-works/system-information/.

[48] R. Zimmerman, C. Murillo-Sanchez, and R. Thomas, "Matpower: Steady-state operations, planning, and analysis tools for power systems research and education," IEEE Trans. Power Syst., vol. 26, no. 1, pp. 12-19, Feb. 2011.

[49] "Simplified 157-bus data," http://www.eee.hku.hk/ yhhou/Simplified\% 20157-bus\%20system\%20data.pdf/.

[50] W. Wei, F. Liu, S. Mei, and Y. Hou, "Robust energy and reserve dispatch under variable renewable generation," IEEE Trans. Smart Grid, vol. 6 , no. 1, pp. 369-380, Jan. 2015.

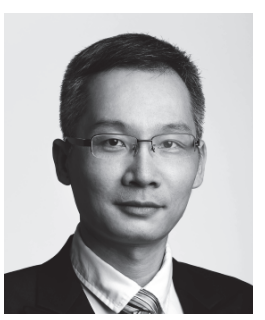

Zhijun Qin (S'11-M'15) received the B.E. and M.S. degrees from Huazhong University of Science and Technology, Wuhan, China, in 2000 and 2003, respectively, and the Ph.D. degree from The University of Hong Kong, Hong Kong, China, in 2015, all in electrical engineering. Currently, he is a post-doctoral research fellow with The University of Hong Kong. Previously, he was a Lecturer with Guangxi University, Nanning, China, from 2004 to 2011. His research interests include power system resilience, renewable energy integration, optima power flow and power system dynamics.

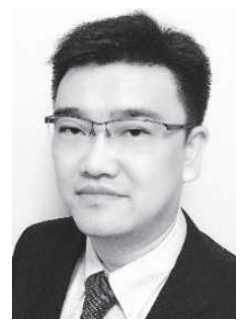

Yunhe Hou (M'08-SM'15) received the B.E. and $\mathrm{Ph} . \mathrm{D}$. degrees in electrical engineering from the Huazhong University of Science and Technology, Wuhan, China, in 1999 and 2005, respectively. He was a Post-Doctoral Research Fellow at Tsinghua University, Beijing, China, from 2005 to 2007, and a Post-Doctoral Researcher at Iowa State University, Ames, IA, USA, and the University College Dublin, Dublin, Ireland, from 2008 to 2009 . He was also a Visiting Scientist at the Laboratory for Information and Decision Systems, Massachusetts Institute of Technology, Cambridge, MA, USA, in 2010. He joined the faculty of the University of Hong Kong, Hong Kong, in 2009, where he is currently an Assistant Professor with the Department of Electrical and Electronic Engineering. Dr. Hou is an Editor of the IEEE TRANSACTIONS ON SMART GRID and an Associate Editor of JOURNAL OF MODERN POWER SYSTEMS AND CLEAN ENERGY.

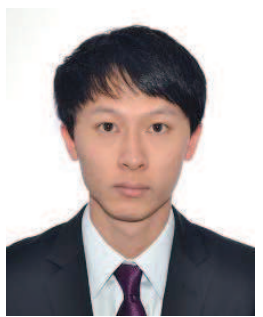

Shunbo Lei (S'14) received his B.E. (2013) degree from Huazhong University of Science and Technology, Wuhan, China. Since 2013, he is a Ph.D. student with The University of Hong Kong, Hong Kong, China. He is currently a Visiting Ph.D. student at Argonne National Laboratory, Argonne, IL, USA. His research interests include optimization of power system operation, renewables integration and power system resilience.

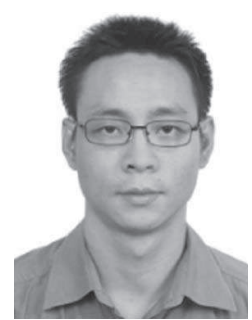

Feng Liu (M'10) received the B.Sc. and Ph.D degrees in electrical engineering from Tsinghua University, Beijing, China, in 1999 and 2004, respectively. He is currently an Associate Professor with Tsinghua University. His research interests include power system analysis and control, and renewable generation. 